\title{
Conventional and microwave assisted synthesis of 1,4-disubstituted 1,2,3-triazoles from Huisgen cycloaddition
}

\author{
Juan I. Sarmiento-Sánchez, Adrián Ochoa-Terán, and Ignacio A. Rivero* \\ Centro de Graduados e Investigación en Química. Instituto Tecnológico de Tijuana, \\ Apartado Postal 1166, Tijuana, B. C. 22000 México \\ E-mail: irivero@tectijuana.mx
}

\begin{abstract}
In this paper the synthesis of a library of new 1,4-disubstituted 1,2,3-triazoles $\mathbf{1}$, with a variety of additional functional groups on its structure, from an in situ generated benzyl azide $\mathbf{2}$ and different alkynes and dialkynes $\mathbf{3}$ is reported. Optimal experimental conditions were established for the conventional click chemistry and for the microwave-assisted synthesis of these 1,2,3triazoles. Comparing the results it was concluded that under microwave-assisted conditions the products are obtained in higher yields in shorter times.
\end{abstract}

Keywords: 1,2,3-Triazoles, microwaves, click chemistry

\section{Introduction}

In recent years, triazole compounds have received much attention due to their wide range of applications in organic and medicinal chemistry. Specifically, 1,2,3-triazoles have been used in pharmaceuticals, agrochemicals, dyes, photographic materials and corrosion inhibitors materials. ${ }^{1}$ There are numerous examples in the literature of the biological activity of triazole compounds as anti-HIV agents $^{2}(\mathbf{4}$ and $\mathbf{5}$ ) or antibiotic agents (6 and 7) due to their antimicrobial activity against Gram positive bacteria, ${ }^{3}$ and as selective $\beta_{3}$ adrenergic agonist receptors (8 and 9). ${ }^{4}$ 
<smiles>[R]c1cn(C2OC(CO[As])C3(OS(=O)(=O)C=C3N)C2O[SnH])nn1</smiles>

$4 \mathrm{R}=\mathrm{CH}\left(\mathrm{OCH}_{2} \mathrm{CH}_{3}\right)_{3}$

$5 \mathrm{R}=\mathrm{CO}_{2} \mathrm{CH}_{3}$<smiles>CNC[C@H]1CN(c2ccc(-n3cc(CO)nn3)c(F)c2)C(=O)O1</smiles>

6

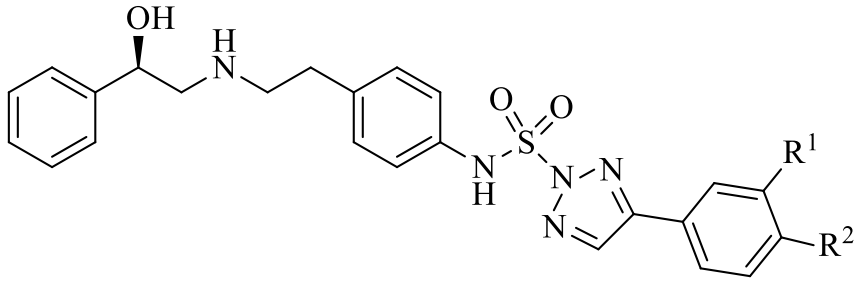

$8 \mathrm{R}^{1}=\mathrm{H} \mathrm{R}^{2}=\mathrm{CF}_{3}$

$9 \mathrm{R}^{1}=\mathrm{F}^{2}=\mathrm{F}$

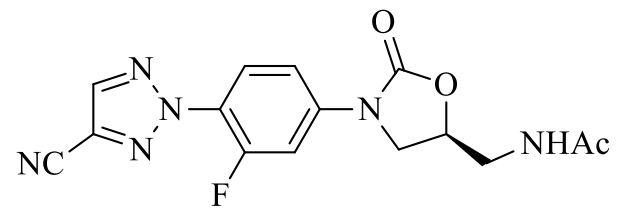

7

Several methods have been described for the synthesis of 1,2,3-triazoles, but commonly they are available from the thermally induced Huisgen cycloaddition reaction between azides and alkynes. ${ }^{5}$ This cycloaddition reaction usually affords mixtures of 1,4- and 1,5-disubstituted 1,2,3triazoles. ${ }^{6}$ Recently, Sharpless ${ }^{7 \mathrm{a}}$ and Meldal $^{7 \mathrm{~b}}$ reported, that 1,4-disubstituted 1,2,3-triazoles are specifically prepared from azides and terminal alkynes under copper(I) catalysis to give 1,4substituted products with high regioselectivity. The regioisomeric 1,5-disubstituted triazoles are available from azides and terminal alkynes by the use of either magnesium acetylides or ruthenium catalysts. ${ }^{8,9}$ In the mechanism proposed by Sharpless for this reaction, the copper(I) ion is inserted into the terminal alkyne, forming the copper(I) acetylide $\mathbf{i}$; this compound reacts with an organic azide and a subsequent rearrangement forms the final product iii (Scheme 1). Because of the existence of copper(I) acetylide $\mathbf{i}$, the reaction was regiospecific and only the 1,4disubstituted 1,2,3-triazole was formed. ${ }^{7 \mathrm{a}}$

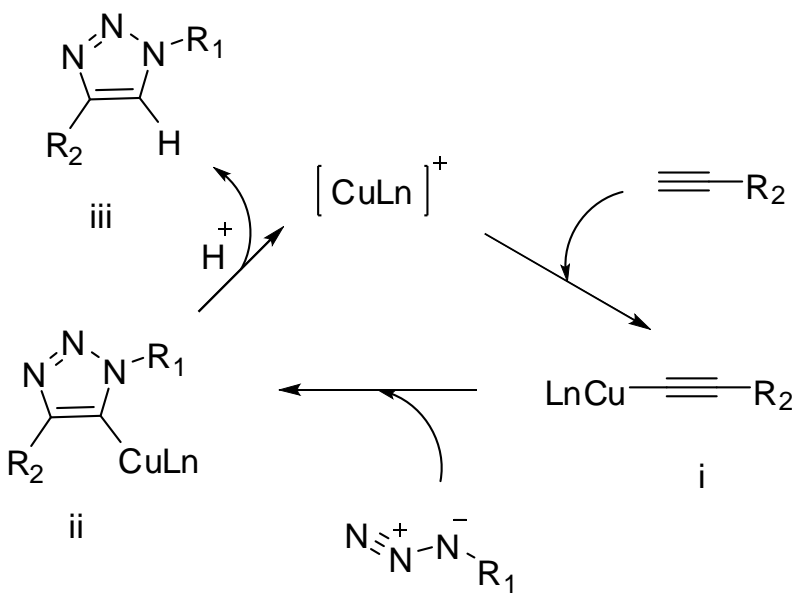

Scheme 1. Mechanism of 1,3-dipolar reaction catalyzed with $\mathrm{Cu}(\mathrm{I})$ salt. 
On the other hand, microwaves have been recognized as a valuable tool for assisting a variety of chemical reactions. ${ }^{10}$ A fast and simple procedure of microwave-assisted synthesis of 1-aryl1,2,3-triazoles from aryl azides and $\alpha$-keto phosphorus ylides on a silica gel support was reported by Lan Tao and co-workers, ${ }^{11}$ and recently, Castagnolo reported the synthesis of new enantiomerically pure triazole derivatives and their evaluation as inhibitors of Mycobacterium tuberculosis. ${ }^{12}$ With this method, propargylamides and organic azides were reacted to generate 1,2,3-triazoles via a microwave-assisted click reaction in the presence of $\mathrm{Cu} / \mathrm{Na}$ ascorbate, to generate the copper(I) catalyst in situ. Furthermore, Van der Eycken and coworkers used microwave-assisted click chemistry for the synthesis of 1,4-disubstituted 1,2,3-triazoles via a copper(I)-catalyst generated in situ by the comproportionation reaction of $\mathrm{Cu}(0)$ and $\mathrm{Cu}(\mathrm{II}) .{ }^{13}$ Fokin and co-workers ${ }^{14}$ developed a method for the synthesis of 1,4-disubstituted 1,2,3-triazoles directly from a variety aryl and vinyl halides using a copper(I)-catalyzed proline-promoted reaction in a mixture of dimethylsulfoxide/water (9:1) or dimethylformamide/water $(4: 1)$ as the solvent, at $60{ }^{\circ} \mathrm{C}$ overnight.

The cycloaddition of azides and alkynes is typically carried out in refluxing toluene, but labile molecules may not be stable under these conditions. Also, although organic azides are generally safe compounds, those of low molecular weight can be unstable and, therefore, difficult to handle. ${ }^{15}$ This is especially true for small molecules with several azide functionalities, which would be of much interest for the generation of polyvalent structures. Thus, a methodology that avoids isolation of organic azides is desirable. In this paper, the synthesis of new 1,4-disubstituted 1,2,3-triazoles from organic azides generated in situ under conventional click chemistry conditions and microwave assisted click conditions is reported.

\section{Results and Discussion}

As depicted in Scheme 2, the first step in the synthesis of triazoles is the in situ formation of an organic azide by the reaction of sodium azide and an alkyl bromide in aqueous tert-butyl alcohol $\left({ }^{t} \mathrm{BuOH} / \mathrm{H}_{2} \mathrm{O} 1: 1 \mathrm{v} / \mathrm{v}\right)$ at $25{ }^{\circ} \mathrm{C}$. In this sense, three reactions were performed simultaneously at controlled temperature using benzyl bromide and sodium azide to establish the time for the completion of this reaction. The three samples were analyzed by high performance liquid chromatography (HPLC) using a methanol-water (7:3) mixture as eluent and UV detection at $214.16 \mathrm{~nm}$. Through these experiments an optimal time of $130 \mathrm{~min}$ to complete these reactions was found, and the organic azide was isolated with $80 \%$ yield. 


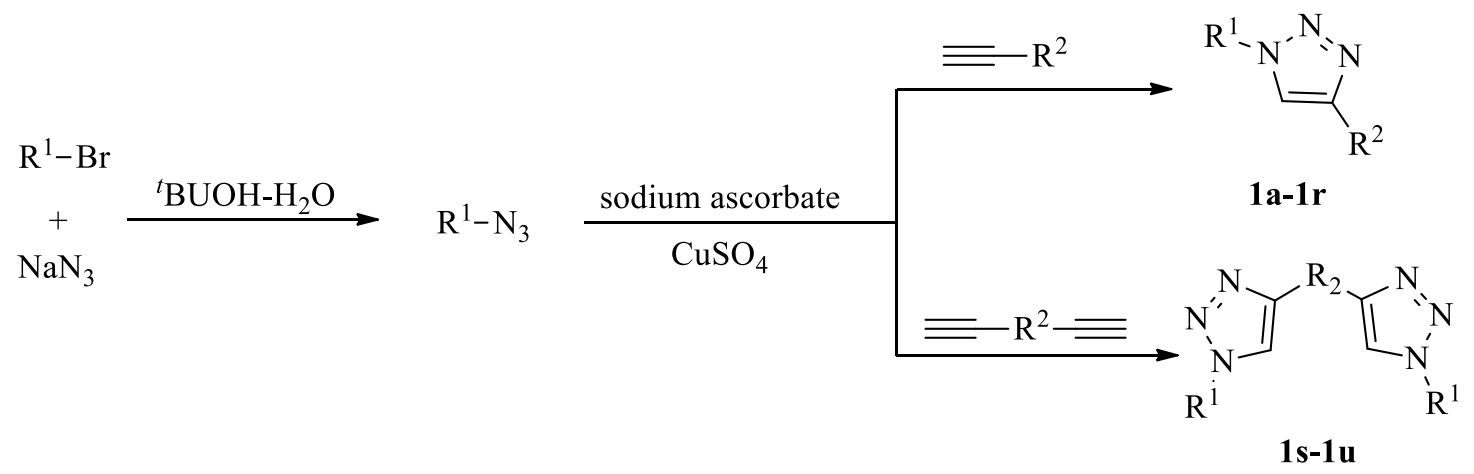

Scheme 2. Synthesis of 1,4-disubstituted 1,2,3-triazoles.

Then, a library of twenty one 1,4-disubstituted 1,2,3-triazoles was synthesized from the thermally induced Huisgen cycloaddition reaction between benzyl azide generated in situ and different alkynes and dialkynes. A temperature of 50 to $60{ }^{\circ} \mathrm{C}$ and a time of 6 to $12 \mathrm{~h}$ were the conditions for the synthesis of most of the products. The isolated product yields were in the range of 37 to $98 \%$, depending of the alkyne used as starting material. Is noteworthy that in the case of products $1 \mathbf{i}, \mathbf{1 k}, \mathbf{1}$ and $\mathbf{1 m}$, it was necessary to prepare the benzyl azide at $60{ }^{\circ} \mathrm{C}$ and after two hours to add the corresponding alkyne, sodium ascorbate and copper (II) sulfate, in order to prevent the formation of byproducts and increase the yield of the isolated product. Also, a different behavior was observed with the alkyne 3,3-diethoxypropyne under different reaction conditions (Table 1, entries 1 and 2): when the reaction was performed at room temperature for $12 \mathrm{~h}$ the acetal triazole 1a was exclusively obtained, whereas at higher temperatures the isolated product was the formyl triazole 1b. Acidic conditions are normally required for the hydrolysis of acetal groups; in this case it seems to be a mild acid condition generated by ascorbic acid and copper(II) ions present in the reaction mixture, which is enough to hydrolyze the acetal group to the aldehyde at higher temperature.

As is shown in Table 1, a variety of alkynes were used in the synthesis, which allowed the inclusion of different functional groups to the triazole molecules. It is interesting to found that the yield for the hydroxyalkyltriazoles 1c-1f decreases as the chain length increases; this might be due to the hydrophobicity of larger hydrocarbon chain compounds. Also, in the case of the anilinyl triazoles $\mathbf{1 k - 1 m}$, there are dramatic differences on the yield, which increases as the amino group is separated from the triazole group. This is a clear evidence of a steric, more than an electronic effect on the reaction process. 


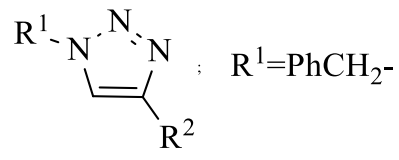

Table 1. 1,4-Disubstituted-1,2,3-triazoles synthesized

\begin{tabular}{|c|c|c|c|c|}
\hline Entry & Product & $\mathrm{R}^{2}$ & Yield $(\%)^{\mathrm{a}}$ & $\begin{array}{l}\text { MW } \\
\text { Yield } \\
(\%)^{\mathrm{b}}\end{array}$ \\
\hline 1 & $1 \mathbf{a}$ & $-\mathrm{CH}\left(\mathrm{OCH}_{2} \mathrm{CH}_{3}\right)_{2}$ & $94^{\mathrm{c}}$ & - \\
\hline 2 & $1 b$ & $-\mathrm{CH}=\mathrm{O}$ & 82 & 90 \\
\hline 3 & $1 \mathrm{c}$ & $-\mathrm{CH}_{2} \mathrm{OH}$ & 90 & 91 \\
\hline 4 & 1d & $-\mathrm{CH}_{2} \mathrm{CH}_{2} \mathrm{OH}$ & 87 & 93 \\
\hline 5 & 1e & $-\mathrm{CH}_{2} \mathrm{CH}_{2} \mathrm{CH}_{2} \mathrm{OH}$ & 81 & 88 \\
\hline 6 & 1f & $-\mathrm{CH}_{2} \mathrm{CH}_{2} \mathrm{CH}_{2} \mathrm{CH}_{2} \mathrm{OH}$ & 82 & 85 \\
\hline 7 & $1 g$ & $-\mathrm{CH}(\mathrm{OH}) \mathrm{CH}_{3}$ & 93 & - \\
\hline 8 & $1 \mathrm{~h}$ & $-\mathrm{CH}_{2} \mathrm{CH}(\mathrm{OH}) \mathrm{CH}_{3}$ & 68 & 80 \\
\hline 9 & $\mathbf{1 i}$ & $-\mathrm{CH}_{2} \mathrm{Cl}$ & 51 & 48 \\
\hline 10 & $\mathbf{1 j}$ & 3-hydroxyphenyl & 77 & 52 \\
\hline 11 & $1 \mathrm{k}$ & 2-aminophenyl & 42 & 42 \\
\hline 12 & 11 & 3-aminophenyl & 65 & - \\
\hline 13 & $1 \mathrm{~m}$ & 4-aminophenyl & 86 & 32 \\
\hline 14 & 1n & 1-naphthyl & 85 & - \\
\hline 15 & 10 & $-\mathrm{C}\left(\mathrm{CH}_{3}\right)_{2} \mathrm{NH}_{2}$ & 82 & - \\
\hline 16 & $1 p$ & 1-hydroxy-c-pentyl & 98 & - \\
\hline 17 & $1 q$ & 1-hydroxy-c-hexyl & 77 & - \\
\hline 18 & $1 \mathbf{r}$ & $-\mathrm{CH}=\mathrm{O}$ & 71 & - \\
\hline 19 & $1 \mathrm{~s}$ & $-\mathrm{CH}_{2} \mathrm{OCH}_{2}-$ & 38 & 37 \\
\hline 20 & 1t & 1,3-phenylen & 40 & 45 \\
\hline 21 & $1 \mathbf{u}$ & $-\mathrm{CH}_{2} \mathrm{CH}_{2} \mathrm{CH}_{2-}$ & 81 & - \\
\hline
\end{tabular}

On the other hand, the microwave-assisted click synthesis of triazole compounds started with the optimization of reaction conditions. For this purpose the synthesis of product 1c was studied. First, in accordance with the literature, the time of reaction was set at 12 min and then, varying the temperature from $50{ }^{\circ} \mathrm{C}$ to $130{ }^{\circ} \mathrm{C}$, it was found that at lower temperatures the product is not formed or very poor yields are obtained. In contrast, at higher temperatures $\left(120^{\circ} \mathrm{C}\right.$ and $\left.130{ }^{\circ} \mathrm{C}\right)$, quantitative yields are obtained (Figure 1a). Then, keeping the temperature at $120{ }^{\circ} \mathrm{C}$, the reaction was performed at different times, obtaining the maximum yield at $12 \mathrm{~min}$. (Figure 1b). Through these experiments the optimal reaction conditions at $120{ }^{\circ} \mathrm{C}$ and 12 min were established, reaching a total conversion of the organic azide to the triazole compound and the isolation of the desired product with excellent yields. The same conditions were used for the 
synthesis of other triazole compounds previously prepared under conventional conditions, obtaining comparable or higher yields in the same time (Table 1).
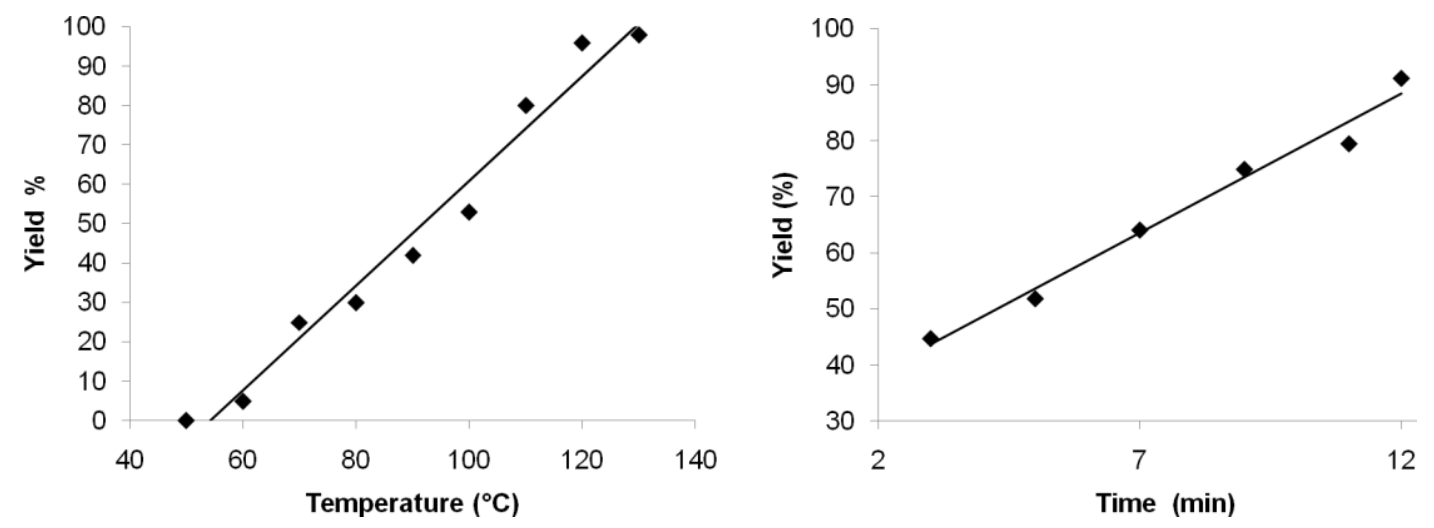

Figure 1. Optimization of the click chemistry microwave assisted conditions for 1c compound.

The yields obtained for triazole compounds prepared using monofunctional alkynes microwave-assisted click conditions were higher than those obtained under conventional conditions. Also, this technique has the advantage of being simple and allows the synthesis of the triazole compounds on a minimum of time. From an experimental point of view, the microwaveassisted click synthesis required only the reagents and the microwave irradiation. The isolation of the products was accomplished either by filtration of the reaction mixture through a celite and silica gel pad, or by extraction with ethyl acetate followed by purification by flash chromatography for some of the products.

In case of polyfunctional substrates as $\mathbf{3 j}$, 3k and $\mathbf{3 m}$ the yields were lower than those obtained under thermal conditions. When crude products were purified the $O$ - or $N$-alkylated derivatives were isolated as mainly products. For dialkynes $\mathbf{3 s}$ and $\mathbf{3 t}$ yields were comparable in both methods.

\section{Conclusions}

In this work a methodology was developed for the synthesis of 1,4-disubstituted 1,2,3- triazole compounds in excellent yields from an in situ generated alkyl azide and different alkynes. Also, it was found that the microwave irradiation dramatically reduces the reaction times from hours to several minutes, which is an important factor on the viability of new synthetic methods. In addition, the products are obtained in higher or comparable yields with those obtained under conventional thermal conditions. 


\section{Experimental Section}

General. All reagents were purchased in the highest quality available and were used without further purification. The solvents used in column chromatography were obtained from commercial suppliers and used without distillation. Infrared spectra (FTIR) were recorded on a Perkin Elmer FT-IR 1600 spectrophotometer. Nuclear magnetic resonance ${ }^{1} \mathrm{H}$ (at $200 \mathrm{MHz}$ ) and ${ }^{13} \mathrm{C}$ (at $50 \mathrm{MHz}$ ) spectra were recorded on a Varian Mercury $200 \mathrm{MHz}$ Spectrometer in $\mathrm{CDCl}_{3}$ with TMS as internal standard. Electrospray ionization mass spectra (ESI-MS) were obtained with an ion trap, and the intensities are reported as a percentage relative to the base peak after the corresponding $\mathrm{m} / \mathrm{z}$ value. Melting points were obtained on an Electrothermal 88629 apparatus. All of the reactions under microwave irradiation were conducted in heavy-walled Pyrex tubes sealed with aluminum crimp caps fitted with a silicon septum. Microwave heating was carried out with a single mode cavity Discover microwave synthesizer (CEM Corp.) producing continuous irradiation at $2455 \mathrm{MHz}$.

General procedure for the synthesis of 1,4-disubstituted-1,2,3-triazoles under microwaveassisted conditions

To a solution ${ }^{t} \mathrm{BuOH} / \mathrm{H}_{2} \mathrm{O}(2 \mathrm{~mL} 1: 1 \mathrm{v} / \mathrm{v})$ was added benzyl bromide $(150 \mu \mathrm{L}, 1.261 \mathrm{mmol})$, sodium azide $(82.0 \mathrm{mg}, 1.261 \mathrm{mmol})$, alkyne $(1.261 \mathrm{mmol})$, copper(II) sulfate $(15.7 \mathrm{mg}, 0.063$ $\mathrm{mmol}, 5 \% \mathrm{~mol})$ and sodium ascorbate $(25.0 \mathrm{mg}, 0.13 \mathrm{mmol}, 10 \% \mathrm{~mol})$. The mixture was placed in a microwave reactor vessel $(10 \mathrm{~mL})$ and heated at $120{ }^{\circ} \mathrm{C}$ for $12 \mathrm{~min}$, and then cooled to room temperature. The reaction mixture was filtered with zeolite and silica gel to vacuo, was extracted with ethyl acetate $(40 \mathrm{~mL})$. After evaporation of the solvent, the residual oil was purified by flash chromatography.

\section{General procedure for the synthesis of 1,4-disubtituted-1,2,3-triazoles under thermal conditions}

To a solution ${ }^{t} \mathrm{BuOH} / \mathrm{H}_{2} \mathrm{O}(6 \mathrm{~mL} 1: 1 \mathrm{v} / \mathrm{v})$ was added alkyl bromide $(200 \mu \mathrm{L}, 1.680 \mathrm{mmol})$, sodium azide (109.5 mg, $1.680 \mathrm{mmol})$, alkyne $(1.680 \mathrm{mmol})$, copper(II) sulfate (21 $\mathrm{mg}, 0.080$ $\mathrm{mmol}, 5 \% \mathrm{~mol})$ and sodium ascorbate $(33.4 \mathrm{mg}, 0.170 \mathrm{mmol}, 10 \% \mathrm{~mol})$ with vigorous stirring at 50-60 ${ }^{\circ} \mathrm{C}$ for 6-12 h. The reaction mixture was filtered with diatomaceous earth or zeolite and silica gel in vacuo, then extracted with ethyl acetate $(80 \mathrm{~mL})$. The extracts were combined and dried over anhydrous sodium sulphate. After evaporation of the solvent, the residual oil was purified by flash chromatography.

1-Benzyl-4-(diethoxymethyl)-1H-1,2,3-triazole (1a). Yield 94\%; white solid; mp 56-57 ${ }^{\circ} \mathrm{C}$ (lit. $\left.{ }^{16} 58-60{ }^{\circ} \mathrm{C}\right) ;{ }^{1} \mathrm{H}$ NMR $\left(\mathrm{CDCl}_{3}, 200 \mathrm{MHz}\right): \delta 7.50(\mathrm{~s}, 1 \mathrm{H}), 7.40-7.24(\mathrm{~m}, 5 \mathrm{H}), 5.70(\mathrm{~s}, 1 \mathrm{H})$, $5.52(\mathrm{~s}, 2 \mathrm{H}), 3.75-3.50(\mathrm{~m}, 4 \mathrm{H}), 1.22(\mathrm{t}, J=7.05 \mathrm{~Hz}, 6 \mathrm{H}) ;{ }^{13} \mathrm{C} \mathrm{NMR}\left(\mathrm{CDCl}_{3}, 50 \mathrm{MHz}\right): \delta 147.5$, 134.5, 129.1, 128.8, 128.2, 121.8, 96.8, 61.7, 54.2, 15.1; IR (KBr): 1653, 1601, 1458, $1096 \mathrm{~cm}^{-1}$. ESI-MS m/z: $284[\mathrm{M}+\mathrm{Na}]^{+}, 300[\mathrm{M}+\mathrm{K}]^{+}, 545[2 \mathrm{M}+\mathrm{Na}]^{+}$. 
1-Benzyl-1H-1,2,3-triazole-4-carbaldehyde (1b). Yield 82\%; white solid; mp 68-70 ${ }^{\circ} \mathrm{C}$ (lit. ${ }^{17}$ 88-90 $\left.{ }^{\circ} \mathrm{C}\right) ;{ }^{1} \mathrm{H}$ NMR $\left(\mathrm{CDCl}_{3}, 200 \mathrm{MHz}\right): \delta 10.11(\mathrm{~s}, 1 \mathrm{H}), 8.04(\mathrm{~s}, 1 \mathrm{H}), 7.40-7.30(\mathrm{~m}, 5 \mathrm{H}), 5.60(\mathrm{~s}$, $2 \mathrm{H}) ;{ }^{13} \mathrm{C} \mathrm{NMR}\left(\mathrm{CDCl}_{3}, 50 \mathrm{MHz}\right): \delta 185.0,147.9,133.4,129.3,129.2,128.3,125.2$, 54.5; IR $(\mathrm{KBr}): \quad 2853, \quad 1691, \quad 1604, \quad 1448, \quad \mathrm{~cm}^{-1} ; \quad$ ESI-MS $m / z: 242 \quad[\mathrm{M}+\mathrm{MeOH}+\mathrm{Na}]^{+}, 461$ $[2 \mathrm{M}+\mathrm{MeOH}+\mathrm{Na}]^{+}$.

(1-Benzyl-1H-1,2,3-triazol-4-yl)methanol (1c). Yield 90\%; pale yellow solid; mp 75-76 ${ }^{\circ} \mathrm{C}$, (lit. $\left.{ }^{16,18,19 \mathrm{c}} 76-78{ }^{\circ} \mathrm{C}\right) .{ }^{1} \mathrm{H}$ NMR $\left(\mathrm{CDCl}_{3}, 200 \mathrm{MHz}\right): \delta 7.45(\mathrm{~s}, 1 \mathrm{H}), 7.37-7.22(\mathrm{~m}, 5 \mathrm{H}), 5.48$ (s, 2H), $4.76(\mathrm{~s}, 2 \mathrm{H}) ;{ }^{13} \mathrm{C} \mathrm{NMR}\left(\mathrm{CDCl}_{3}, 50 \mathrm{MHz}\right): \delta 148.2,134.5,129.2,128.8,128.1,121.7,56.3$, 54.2; IR (KBr): 3265, 1678, 1605, 1455, $1221 \mathrm{~cm}^{-1}$. ESI-MS m/z: $212[\mathrm{M}+\mathrm{Na}]^{+}, 400[2 \mathrm{M}+\mathrm{Na}]^{+}$. 2-(1-Benzyl-1H-1,2,3-triazol-4-yl)ethanol (1d). Yield 87\%; pale yellow oil (lit. ${ }^{13,19}$ ); ${ }^{1} \mathrm{H}$ NMR $\left(\mathrm{CDCl}_{3}, 200 \mathrm{MHz}\right): \delta 7.40-7.24(\mathrm{~m}, 6 \mathrm{H}), 5.50(\mathrm{~s}, 2 \mathrm{H}), 3.95-3.89(\mathrm{t}, J=5.89 \mathrm{~Hz}, 2 \mathrm{H}), 2.94-2.88$ $(\mathrm{t}, J=5.77 \mathrm{~Hz}, 2 \mathrm{H}) ;{ }^{13} \mathrm{C}-\mathrm{NMR}\left(\mathrm{CDCl}_{3}, 50 \mathrm{MHz}\right): \delta 145.9,134.6,129.1,128.7,128.0,121.7$, 61.3, 54.0, 28.7; IR (film): 3357, 1653, 1601, $1454 \mathrm{~cm}^{-1}$. ESI-MS m/z: $204[\mathrm{M}+\mathrm{H}]^{+}, 226$ $[\mathrm{M}+\mathrm{Na}]^{+}, 242[\mathrm{M}+\mathrm{K}]^{+}, 429[2 \mathrm{M}+\mathrm{Na}]^{+}$.

3-(1-Benzyl-1H-1,2,3-triazol-4-yl)propan-1-ol (1e). Yield 81\%; white solid; mp 42-43 ${ }^{\circ} \mathrm{C}$. $R_{f}$ 0.43 (petroleum ether/EtOAc 1:1 v/v); ${ }^{1} \mathrm{H} \mathrm{NMR}\left(\mathrm{CDCl}_{3}, 200 \mathrm{MHz}\right): \delta 7.33(\mathrm{~m}, 6 \mathrm{H}), 5.47(\mathrm{~s}$, 2H), 3.87 (br s, 1H), 3.65 (t, 2H, $J=6.16 \mathrm{~Hz}$ ), $2.78(\mathrm{t}, 2 \mathrm{H}, J=7.34 \mathrm{~Hz}), 1.88\left(\mathrm{q}, 2 \mathrm{H}, J_{1}=6.28\right.$ $\left.\mathrm{Hz}, J_{2}=13.51 \mathrm{~Hz}\right) ;{ }^{13} \mathrm{C} \mathrm{NMR}\left(\mathrm{CDCl}_{3}, 50 \mathrm{MHz}\right): \delta 148.0,134.7,129.0,128.6,127.9,121.0,61.4$, 54.0, 32.0, 21.9; IR (KBr): 3356, 1656, 1605, 1453, $1054 \mathrm{~cm}^{-1}$. ESI-MS $m / z: 218[\mathrm{M}+\mathrm{H}]^{+}$.

4-(1-Benzyl-1H-1,2,3-triazol-4-yl)butan-1-ol (1f). Yield 82\%; white solid; mp 58-59 ${ }^{\circ} \mathrm{C}$ (lit. $^{20}$ $\left.80{ }^{\circ} \mathrm{C}\right) ;{ }^{1} \mathrm{H} \mathrm{NMR}\left(\mathrm{CDCl}_{3}, 200 \mathrm{MHz}\right): \delta$ 7.40-7.22 (m, 6H), $5.48(\mathrm{~s}, 2 \mathrm{H}), 3.65$ (t, $J=6.18 \mathrm{~Hz}$, 2H), $2.73(\mathrm{t}, J=6.93 \mathrm{~Hz}, 2 \mathrm{H}), 1.80-1.54(\mathrm{~m}, 4 \mathrm{H}) ;{ }^{13} \mathrm{C} \mathrm{NMR}\left(\mathrm{CDCl}_{3}, 50 \mathrm{MHz}\right): \delta 148.5,134.8$, 129.0, 128.6, 127.9, 120.7, 62.1, 53.9, 32.1, 25.5, 25.2; IR (KBr): 3387, 1653, 1601, 1458, 1214 $\mathrm{cm}^{-1}$. ESI-MS m/z: $232[\mathrm{M}+\mathrm{H}]^{+}, 254[\mathrm{M}+\mathrm{Na}]^{+}, 270[\mathrm{M}+\mathrm{K}]^{+}, 485[2 \mathrm{M}+\mathrm{Na}]^{+}$.

(S)-1-(1-Benzyl-1H-1,2,3-triazol-4-yl)etanol (1g). Yield 93\%; white solid; mp 64-65 ${ }^{\circ} \mathrm{C}$ (lit. ${ }^{21}$ ); ${ }^{1} \mathrm{H} \mathrm{NMR}\left(\mathrm{CDCl}_{3}, 200 \mathrm{MHz}\right): \delta 7.40-7.25(\mathrm{~m}, 6 \mathrm{H}), 5.50(\mathrm{~s}, 2 \mathrm{H}), 5.10-5.00\left(\mathrm{q}, J_{l}=6.49 \mathrm{~Hz}, J_{2}=\right.$ $12.98 \mathrm{~Hz}, 1 \mathrm{H}), 1.55(\mathrm{~d}, J=6.56 \mathrm{~Hz}, 3 \mathrm{H}) ;{ }^{13} \mathrm{C} \mathrm{NMR}\left(\mathrm{CDCl}_{3}, 50 \mathrm{MHz}\right): \delta 152.9,134.5,129.1$, 128.8, 128.1, 120.2, 62.8, 54.1, 23.1; IR (KBr): 3357, 1653, 1587, 1498, 1458, $1292 \mathrm{~cm}^{-1}$. ESI$\mathrm{MS} m / z: 226[\mathrm{M}+\mathrm{Na}]^{+}, 429[2 \mathrm{M}+\mathrm{Na}]^{+}$.

1-(1-Benzyl-1H-1,2,3-triazol-4-yl)propan-2-ol (1h). Yield 68\%; white solid; mp 44-45 ${ }^{\circ} \mathrm{C}, R_{f}$ 0.48 (petroleum ether/EtOAc 4:6 v/v); ${ }^{1} \mathrm{H}$ NMR $\left(\mathrm{CDCl}_{3}, 200 \mathrm{MHz}\right): \delta 7.40-7.23(\mathrm{~m}, 6 \mathrm{H}), 5.50$ (s, 2H), 4.21-4.06 (m, 1H), 2.91-2.81 (dd, $J=4.0,15.21 \mathrm{~Hz}, 1 \mathrm{H}), 2.79-2.67(\mathrm{dd}, J=7.70,15.21$ $\mathrm{Hz}, 1 \mathrm{H}), 1.25(\mathrm{~d}, J=6.22 \mathrm{~Hz}, 3 \mathrm{H}) ;{ }^{13} \mathrm{C} \mathrm{NMR}\left(\mathrm{CDCl}_{3}, 50 \mathrm{MHz}\right): \delta 145.7,134.7,129.1,128.7$, 128.0, 121.7, 67.1, 54.1, 34.8, 22.9; IR (KBr): 3387, 1653, 1587, 1458, $1295 \mathrm{~cm}^{-1}$. ESI-MS m/z: $218[\mathrm{M}+\mathrm{H}]^{+}, 240[\mathrm{M}+\mathrm{Na}]^{+}, 256[\mathrm{M}+\mathrm{K}]^{+}, 456[2 \mathrm{M}+\mathrm{Na}]^{+}$. HRMS: calcd. for $\left[\mathrm{C}_{12} \mathrm{H}_{15} \mathrm{~N}_{3} \mathrm{O}+\mathrm{H}\right]^{+}$ 218.1288; found 218.1291 .

1-Benzyl-4-(chloromethyl)-1H-1,2,3-triazole (1i). Yield 51\%; white solid; mp 112-113 ${ }^{\circ} \mathrm{C}$ (lit. ${ }^{18} 114-116{ }^{\circ} \mathrm{C}$ ); $R_{f} 0.27$ (petroleum ether/EtOAc 8:2 v/v); ${ }^{1} \mathrm{H} \mathrm{NMR}\left(\mathrm{CDCl}_{3}, 200 \mathrm{MHz}\right): \delta 7.51$ $(\mathrm{s}, 1 \mathrm{H}), 7.39-7.24(\mathrm{~m}, 5 \mathrm{H}), 5.51(\mathrm{~s}, 2 \mathrm{H}), 4.66(\mathrm{~s}, 2 \mathrm{H}) .{ }^{13} \mathrm{C} \mathrm{NMR}\left(\mathrm{CDCl}_{3}, 50 \mathrm{MHz}\right): \delta 145.0$, 
134.3, 129.2, 128.9, 128.1, 122.6, 54.3, 36.2; IR (KBr): 1653, 1601, 1458, $702 \mathrm{~cm}^{-1}$; ESI-MS $m / z: 208[\mathrm{M}+\mathrm{H}]^{+}, 230[\mathrm{M}+\mathrm{Na}]^{+}, 437[2 \mathrm{M}+\mathrm{Na}]^{+}$.

3-(1-Benzyl-1H-1,2,3-triazol-4-yl)phenol (1j). Yield 77\%; white solid; mp 130-131 ${ }^{\circ} \mathrm{C} ;{ }^{1} \mathrm{H}$ NMR $\left(\mathrm{CDCl}_{3}, 200 \mathrm{MHz}\right): \delta 8.96(\mathrm{~s}, 1 \mathrm{H}), 7.72(\mathrm{~s}, 1 \mathrm{H}), 7.36-7.17(\mathrm{~m}, 7 \mathrm{H}), 6.84-6.78\left(\mathrm{ddd}, J_{1}=\right.$ $\left.6.57 \mathrm{~Hz}, J_{2}=2.25 \mathrm{~Hz}, J_{3}=2.17 \mathrm{~Hz}, 1 \mathrm{H}\right), 5.55(\mathrm{~s}, 2 \mathrm{H}) ;{ }^{13} \mathrm{C}-\mathrm{NMR}\left(\mathrm{CDCl}_{3}, 50 \mathrm{MHz}\right): \delta 157.7$, 148.1, 134.8, 131.6, 129.8, 129.0, 128.6, 127.9, 119.8, 116.7, 115.5, 112.7, 54.0; IR (KBr): 3148, 1621, 1587, 1457, $1217 \mathrm{~cm}^{-1}$; ESI-MS m/z: $252[\mathrm{M}+\mathrm{H}]^{+}, 274[\mathrm{M}+\mathrm{Na}]^{+}, 290[\mathrm{M}+\mathrm{K}]^{+}, 525$ $[2 \mathrm{M}+\mathrm{Na}]^{+}$. HRMS: calcd. for $\left[\mathrm{C}_{15} \mathrm{H}_{13} \mathrm{~N}_{3} \mathrm{O}+\mathrm{H}\right]^{+}$252.1131; found 252.1139 .

2-(1-Benzyl-1H-1,2,3-triazol-4-yl)aniline (1k). Yield 42\%; pale yellow solid, m.p. 97-98 ${ }^{\circ} \mathrm{C} ; R_{f}$ 0.25 (petroleum ether/EtOAc 4:6 v/v); ${ }^{1} \mathrm{H}$ NMR $\left(\mathrm{CDCl}_{3}, 200 \mathrm{MHz}\right): \delta 7.65(\mathrm{~s}, 1 \mathrm{H}), 7.40-7.24$ $(\mathrm{m}, 6 \mathrm{H}), 7.12-7.04\left(\mathrm{dd}, J_{1}=8.04, J_{2}=1.55 \mathrm{~Hz}, 1 \mathrm{H}\right), 6.76-6.62(\mathrm{~m}, 2 \mathrm{H}), 5.55(\mathrm{~s}, 2 \mathrm{H}), 5.50(\mathrm{br} \mathrm{s}$, $2 \mathrm{H}) ;{ }^{13} \mathrm{C} \mathrm{NMR}\left(\mathrm{CDCl}_{3}, 50 \mathrm{MHz}\right): \delta 148.8,145.1,134.5,129.1,129.0,128.7,128.0,127.6$, 119.7, 117.2, 116.7, 113.5, 54.3; IR (KBr): 3343, 1653, 1601, $1454 \mathrm{~cm}^{-1}$; ESI-MS m/z: 251 $[\mathrm{M}+\mathrm{H}]^{+}, 273[\mathrm{M}+\mathrm{Na}]^{+}, 523[2 \mathrm{M}+\mathrm{Na}]^{+}$. HRMS: calcd. for $\left[\mathrm{C}_{15} \mathrm{H}_{14} \mathrm{~N}_{4}+\mathrm{H}\right]^{+}$251.1291; found 251.1298 .

3-(1-Benzyl-1H-1,2,3-triazol-4-yl)aniline (11). Yield 65\%; pale brown solid; mp 145-147 ${ }^{\circ} \mathrm{C}$ (lit. ${ }^{22} 149{ }^{\circ} \mathrm{C}$ ); $R_{f} 0.23$ (petroleum ether/EtOAc 8:2 v/v); ${ }^{1} \mathrm{H}$ NMR $\left(\mathrm{CDCl}_{3}, 200 \mathrm{MHz}\right): \delta 7.62$ (s, $1 \mathrm{H}), 7.34-7.22(\mathrm{~m}, 6 \mathrm{H}), 7.10-7.02(\mathrm{t}, J=8.5 \mathrm{~Hz}, 1 \mathrm{H}), 6.73-6.61(\mathrm{~m}, 2 \mathrm{H}), 5.49(\mathrm{~s}, 2 \mathrm{H}) ;{ }^{13} \mathrm{C} \mathrm{NMR}$ $\left(\mathrm{CDCl}_{3}, 50 \mathrm{MHz}\right): \delta 148.8,145.1,134.6,129.1,129.0,128.7,128.0,127.7,119.8,117.2,116.7$, 113.5, 54.3; IR (KBr): 3328, 1657, 1601, $1450 \mathrm{~cm}^{-1}$; ESI-MS m/z: $251[\mathrm{M}+\mathrm{H}]^{+}, 273[\mathrm{M}+\mathrm{Na}]^{+}$, $523[2 \mathrm{M}+\mathrm{Na}]^{+}$.

4-(1-Benzyl-1H-1,2,3-triazol-4-yl)aniline (1m). Yield 86\%; pale brown solid; mp 160-161 ${ }^{\circ} \mathrm{C}$ [lit. ${ }^{23}$ ]; $R_{f} 0.24$ (petroleum ether/EtOAc $\left.1: 1 \mathrm{v} / \mathrm{v}\right) ;{ }^{1} \mathrm{H}$ NMR $\left(\mathrm{CDCl}_{3}, 200 \mathrm{MHz}\right): \delta 7.61-7.57(\mathrm{~d}, J$ $=8.43 \mathrm{~Hz}, 2 \mathrm{H}), 7.52(\mathrm{~s}, 1 \mathrm{H}), 7.38-7.26(\mathrm{~m}, 5 \mathrm{H}), 6.72-6.68(\mathrm{~d}, J=8.43 \mathrm{~Hz}, 2 \mathrm{H}), 5.55(\mathrm{~s}, 2 \mathrm{H}) ;{ }^{13} \mathrm{C}$ NMR $\left(\mathrm{CDCl}_{3}, 50 \mathrm{MHz}\right): \delta 146.5,134.9,130.7,129.0,128.7,128.0,126.9,120.9,118.2,115.2$, 54.2; IR (KBr): 3350, 1625, 1611, $1461 \mathrm{~cm}^{-1}$; ESI-MS $m / z: 251[\mathrm{M}+\mathrm{H}]^{+}, 273[\mathrm{M}+\mathrm{Na}]^{+}, 289$ $[\mathrm{M}+\mathrm{K}]^{+}, 523[2 \mathrm{M}+\mathrm{Na}]^{+}$.

1-Benzyl-4-(naphthalen-1-yl)-1H-1,2,3-triazole (1n). Yield 85\%; pale yellow solid; mp 89-90 ${ }^{\circ} \mathrm{C} ;{ }^{1} \mathrm{H}-\mathrm{NMR}\left(\mathrm{CDCl}_{3}, 200 \mathrm{MHz}\right): \delta 8.39-8.34(\mathrm{~m}, 1 \mathrm{H}), 7.88-7.82(\mathrm{~m}, 2 \mathrm{H}), 7.71(\mathrm{~s}, 1 \mathrm{H}), 7.69-$ $7.66(\mathrm{~d}, J=7.33 \mathrm{~Hz}, 1 \mathrm{H}), 7.52-7.47(\mathrm{dd}, J=6.42,3.48 \mathrm{~Hz}, 4 \mathrm{H}), 7.37-7.36(\mathrm{~d}, J=1.83 \mathrm{~Hz}, 4 \mathrm{H})$, $5.61(\mathrm{~s}, 2 \mathrm{H}) ;{ }^{13} \mathrm{C}-\mathrm{NMR}\left(\mathrm{CDCl}_{3}, 50 \mathrm{MHz}\right): \delta 147.3,134.6,133.8,131.0,129.1,128.8,128.7$, 128.3, 128.0, 127.1, 126.5, 125.9, 125.4, 125.2, 122.4, 54.1; IR (KBr): 1686, 1601, $1454 \mathrm{~cm}^{-1}$; ESI-MS m/z: $286[\mathrm{M}+\mathrm{H}]^{+}, 308[\mathrm{M}+\mathrm{Na}]^{+}, 324[\mathrm{M}+\mathrm{K}]^{+}, 593[2 \mathrm{M}+\mathrm{Na}]^{+}$. HRMS: calcd. for $\left[\mathrm{C}_{19} \mathrm{H}_{15} \mathrm{~N}_{3}+\mathrm{H}\right]^{+}$286.1339; found 286.1345 .

2-(1-Benzyl-1H-1,2,3-triazol-4-yl)propan-2-amine (10). Yield 82\%; pale brown solid; mp 32$34{ }^{\circ} \mathrm{C} ;{ }^{1} \mathrm{H}$ NMR $\left(\mathrm{CDCl}_{3}, 200 \mathrm{MHz}\right): \delta 7.34(\mathrm{~m}, 6 \mathrm{H}), 5.48(\mathrm{~s}, 2 \mathrm{H}), 3.44\left(\mathrm{br} \mathrm{s}, 2 \mathrm{H}, \mathrm{NH}_{2}\right), 1.56(\mathrm{~s}$, $6 \mathrm{H}) ;{ }^{13} \mathrm{C} \mathrm{NMR}\left(\mathrm{CDCl}_{3}, 50 \mathrm{MHz}\right): \delta 153.7,134.6,129.1,128.6,128.1,120.2,54.1,29.3$; IR (film): 3387, 1668, 1605, $1454 \mathrm{~cm}^{-1}$; ESI-MS m/z: $217[\mathrm{M}+\mathrm{H}]^{+}, 239[\mathrm{M}+\mathrm{Na}]^{+}, 455[2 \mathrm{M}+\mathrm{Na}]^{+}$. HRMS: calcd. for $\left[\mathrm{C}_{12} \mathrm{H}_{16} \mathrm{~N}_{4}+\mathrm{H}\right]^{+} 217.1448$; found 217.1543. 
1-(1-Benzyl-1H-1,2,3-triazol-4-yl)cyclopentanol (1p). Yield 98\%; brown solid; mp 70-72 ${ }^{\circ} \mathrm{C}$; ${ }^{1} \mathrm{H} \mathrm{NMR}\left(\mathrm{CDCl}_{3}, 200 \mathrm{MHz}\right): \delta 7.35(\mathrm{~m}, 6 \mathrm{H}), 5.49$ (s, 2H), 3.07 (br s, $\left.1 \mathrm{H}\right), 2.00(\mathrm{~m}, 8 \mathrm{H}) .{ }^{13} \mathrm{C}$ NMR $\left(\mathrm{CDCl}_{3}, 50 \mathrm{MHz}\right): \delta 154.6,134.6,129.0,128.7,128.2,119.8,78.8,54.2,41.1,23.6$; IR (KBr): 3387, 1653, 1587, 1454, $1214 \mathrm{~cm}^{-1}$; ESI-MS m/z: $244[\mathrm{M}+\mathrm{H}]^{+}, 266[\mathrm{M}+\mathrm{Na}]^{+}, 324$ $[\mathrm{M}+\mathrm{K}]^{+}, 509[2 \mathrm{M}+\mathrm{Na}]^{+}$. HRMS: calcd. for $\left[\mathrm{C}_{14} \mathrm{H}_{17} \mathrm{~N}_{3} \mathrm{O}+\mathrm{H}\right]^{+}$244.1444; found 214.1451.

1-(1-Benzyl-1H-1,2,3-triazol-4-yl)cyclohexanol (1q). Yield 77\%; white solid; mp 96-97 ${ }^{\circ} \mathrm{C}$ $\left(\right.$ lit. $\left.^{24}\right) ; R_{f} 0.27$ (petroleum ether/EtOAc 1:1 v/v); ${ }^{1} \mathrm{H}$ NMR $\left(\mathrm{CDCl}_{3}, 200 \mathrm{MHz}\right): \delta 7.38-7.23$ (m, $6 \mathrm{H}), 5.50(\mathrm{~s}, 2 \mathrm{H}), 2.11-1.75(\mathrm{~m}, 10 \mathrm{H}) ;{ }^{13} \mathrm{C} \mathrm{NMR}\left(\mathrm{CDCl}_{3}, 50 \mathrm{MHz}\right): \delta 156.1,134.7,129.1,128.7$, 128.1, 119.5, 69.5, 54.1, 38.0, 25.3, 21.9; IR (KBr): 3301, 1605, 1585, 1453, $1155 \mathrm{~cm}^{-1}$; ESI-MS $m / z: 258[\mathrm{M}+\mathrm{H}]^{+}, 280[\mathrm{M}+\mathrm{Na}]^{+}, 296[\mathrm{M}+\mathrm{K}]^{+}, 537[2 \mathrm{M}+\mathrm{Na}]^{+}$.

1-Cyclopentyl-1H-1,2,3-triazole-4-carbaldehyde (1r). Yield 71\%; pale yellow solid; mp 132$134{ }^{\circ} \mathrm{C} ;{ }^{1} \mathrm{H} \mathrm{NMR}\left(\mathrm{CDCl}_{3}, 200 \mathrm{MHz}\right): \delta 10.13$ (s, 1H), $8.14(\mathrm{~s}, 1 \mathrm{H}), 5.08-4.95(\mathrm{q}, 1 \mathrm{H}), 2.41-2.26$ (m, 2H), 2.13-1.77 (m, 6H); ${ }^{13} \mathrm{C} \mathrm{NMR}\left(\mathrm{CDCl}_{3}, 50 \mathrm{MHz}\right): \delta$ 185.3, 147.5, 123.8, 62.4, 33.4, 23.9; IR (film): 3130, 1698, 1530, $1454 \quad \mathrm{~cm}^{-1}$; ESI-MS $m / z: 220 \quad[\mathrm{M}+\mathrm{MeOH}+\mathrm{Na}]^{+}, 418$ $[2 \mathrm{M}+\mathrm{MeOH}+\mathrm{Na}]^{+}$. HRMS: calcd. for $\left[\mathrm{C}_{8} \mathrm{H}_{11} \mathrm{~N}_{3} \mathrm{O}+\mathrm{H}\right]^{+} 166.0975$; found 166.0973.

\section{General procedures for the synthesis of Bis-1,4-disubstituted-1,2,3-triazoles}

To a solution ${ }^{t} \mathrm{BuOH} / \mathrm{H}_{2} \mathrm{O}(1: 1)$ was added benzyl bromide $(200 \mu \mathrm{L}, 1.680 \mathrm{mmol})$, sodium azide (109.5 mg, $1.680 \mathrm{mmol}$ ), dialkyne (0.5 equiv.), copper sulfate (21 mg, $0.080 \mathrm{mmol}, 5 \% \mathrm{~mol}$ ) and sodium ascorbate $(33.4 \mathrm{mg}, 0.170 \mathrm{mmol}, 10 \% \mathrm{~mol})$ with vigorous stirring at $50{ }^{\circ} \mathrm{C}$ for overnight. The reaction mixture was filtered with diatomaceous earth and silica gel in vacuo, then extracted with ethyl acetate $(80 \mathrm{~mL})$. The extracts were combined and dried over anhydrous sodium sulfate. After evaporation of the solvent, the residual oil was purified by column chromatography.

4,4'-Oxybis(methylene)bis(1-benzyl-1H-1,2,3-triazole) (1s). Yield 38\%; white solid; mp 128$129{ }^{\circ} \mathrm{C}$ (lit. ${ }^{25} 126-127{ }^{\circ} \mathrm{C}$ ); $R_{f} 0.40$ (petroleum ether/EtOAc 1:1 v/v); ${ }^{1} \mathrm{H}$ NMR $\left(\mathrm{CDCl}_{3}, 200\right.$ $\mathrm{MHz}): \delta 7.47(\mathrm{~s}, 2 \mathrm{H}), 7.37-7.22(\mathrm{~m}, 10 \mathrm{H}), 5.49(\mathrm{~s}, 4 \mathrm{H}) 4.66(\mathrm{~s}, 4 \mathrm{H}) ;{ }^{13} \mathrm{C} \mathrm{NMR}\left(\mathrm{CDCl}_{3}, 50\right.$ $\mathrm{MHz}): \delta 144.9,134.5,129.1,128.7,128.2,122.7,63.6,54.1 ; \mathrm{IR}(\mathrm{KBr}): 1602,1584,1452,1083$ $\mathrm{cm}^{-1} ;$ ESI-MS m/z: $361[\mathrm{M}+\mathrm{H}]^{+}$.

1,3-Bis(1-benzyl-1H-1,2,3-triazol-4-yl)benzene (1t). Yield 40\%; white solid; mp 115-116 ${ }^{\circ} \mathrm{C}$; $R_{f} 0.37$ (petroleum ether/EtOAc 1:1 v/v); ${ }^{1} \mathrm{H} \mathrm{NMR}\left(\mathrm{CDCl}_{3}, 200 \mathrm{MHz}\right): \delta 8.18(\mathrm{t}, J=1.53 \mathrm{~Hz}$, $1 \mathrm{H}), 7.79(\mathrm{~d}, J=1.73 \mathrm{~Hz}, 1 \mathrm{H}), 7.73(\mathrm{~d}, J=1.73 \mathrm{~Hz}, 1 \mathrm{H}), 7.36(\mathrm{~s}, 2 \mathrm{H}), 7.47-7.26(\mathrm{~m}, 11 \mathrm{H}), 5.57$ $(\mathrm{s}, 4 \mathrm{H}) .{ }^{13} \mathrm{C} \mathrm{NMR}\left(\mathrm{CDCl}_{3}, 50 \mathrm{MHz}\right): \delta 147.8,134.5,131.0,129.4,129.2,128.8,128.1,125.3$, 122.8, 119.8, 54.3; IR (KBr): 1602, 1584, $1452 \mathrm{~cm}^{-1}$; ESI-MS m/z: 393 [M+H] $]^{+}$HRMS: calcd. for $\left[\mathrm{C}_{24} \mathrm{H}_{20} \mathrm{~N}_{6}+\mathrm{H}\right]^{+}$393.1822; found 393.1831.

1,3-Bis(1-benzyl-1H-1,2,3-triazol-4-yl)propane (1u). Yield 81\%; white solid; mp 128-129 ${ }^{\circ} \mathrm{C}$ (lit. $\left.{ }^{25 \mathrm{a}} 129-130{ }^{\circ} \mathrm{C}\right) ;{ }^{1} \mathrm{H}$ NMR (DMSO-d, $\left.200 \mathrm{MHz}\right): \delta 7.90(\mathrm{~s}, 2 \mathrm{H}), 7.39-7.25(\mathrm{~m}, 10 \mathrm{H}), 5.53(\mathrm{~s}$, $4 \mathrm{H}), 2.68-2.60(\mathrm{t}, J=7.48 \mathrm{~Hz}, 4 \mathrm{H}), 1.97-1.82\left(\mathrm{q}, J_{l}=8.06 \mathrm{~Hz}, J_{2}=15.35 \mathrm{~Hz}, 2 \mathrm{H}\right) ;{ }^{13} \mathrm{C} \mathrm{NMR}$ (DMSO- $\left.d_{6}, 50 \mathrm{MHz}\right): \delta$ 146.7, 136.2, 128.5, 127.8, 127.6, 121.9, 52.5, 28.6, 24.4; IR (KBr): $1602,1584,1452 \mathrm{~cm}^{-1}$; ESI-MS m/z: $359[\mathrm{M}+\mathrm{H}]^{+}, 381[\mathrm{M}+\mathrm{Na}]^{+}$. 


\section{Acknowledgements}

We gratefully acknowledge support for this project by Consejo Nacional de Ciencia y Tecnología (CONACyT, GRANT No SEP-2004-CO1-47835) and Dirección General de Educación Superior Tecnológica (DGEST) for support this project. Juan Sarmiento thanks to CONACYT for the graduate scholarship.

\section{Reference}

1. Katritzky, A. R.; Rees, C. W.; Scriven, E. F. V. Eds. Comprehensive Heterocyclic Chemistry II Elsevier Science: Oxford, UK, 1996; Vol. 4, pp 1-126.

2. Alvarez, R.; Elazquez, S. V.; San, F.; De Aquaro, S. C.; Perno, C. F.; Karlesson, A. Balzarini, J.; Camarasa, M. J. J. Med. Chem. 1994, 37, 4185.

3. Genin, M. J.; Allwine, D. A.; Anderson, D. J.; Barbachyn, M. R.; Emmert, D. E.; Garmon, S. A.; Graber, D. R.; Grega, K. C.; Hester, J. B.; Hutchinson, D. K.; Morris, J.; Reischer, R. J.; Ford, C. W.; Zurenco, G. E.; Hamel, J. C.; Schaadt, R. D.; Stapertand, D.; Yagi, B. H. J. Med. Chem. 2000, 43, 953.

4. Brockunier, L. L.; Parmee, E. R.; Ok, H. O.; Candelore, M. R.; Cascieri, M. A.; Colwell, L. F.; Deng, L.; Feeney, W. P.; Forest, M. J.; Hom, G. J.; MacIntyre, D. E.; Tota, L.; Wyvratt, M. J.; Fisher, M. H.; Weber, A. E. Bioorg. Med. Chem. Lett. 2000, 10, 2111.

5. Huisgen, R.; Szeimies, G.; Moebius, L. Chem. Ber. 1967, 100, 2494.

6. (a) Huisgen, R. In 1,3-Dipolar Cycloaddition Chemistry; Padwa, A., Ed.; Wiley: New York, 1984; pp 1-176. (b) Lwowski, W. In 1,3-Dipolar Cycloaddition Chemistry; Padwa, A., Ed.; Wiley: New York, 1984; pp 559-651.

7. (a) Rostovtsev, V. V.; Green, L. G.; Fokin, V. V.; Sharpless, K. B. Angew. Chem. Int. Ed. 2002, 41, 2596. (b) Tornøe, C. W.; Christensen, C.; Meldal, M. J. Org. Chem. 2002, 67, 3057.

8. (a) Krasinski, A.; Fokin, V. V.; Sharpless, K. B. Org. Lett. 2004, 6, 1237. (b) Akimova, G. S.; Chistokletov, V. N.; Petrov, A. A. Zh. Org. Khim. 1967, 3, 968. (c) Akimova, G. S.; Chistokletov, V. N.; Petrov, A. A. Zh. Org. Khim. 1967, 3, 2241. (d) Akimova, G. S.; Chistokletov, V. N.; Petrov, A. A. Zh. Org. Khim. 1968, 4, 389.

9. (a) Zhang, L.; Chen, X.; Xue, P.; Sun, H. H. Y.; Williams, I. D.; Sharpless, K. B.; Fokin, V. V.; Jia, G. J. Am. Chem. Soc. 2005, 127, 15998. (b) Rasmussen, L. K.; Boren, B. C.; Fokin, V. V. Org. Lett. 2007, 9, 5337.

10. (a) Soh, C. H.; Chui, W. K.; Lam, Y. J. Comb. Chem. 2006, 8, 464. (b) Katritzky, A. R.; Singh, A. K. J. Org. Chem. 2002, 67, 9077.

11. Tao, L.; Zhang, L.; Shen, S.; Han, X. P. Chinese Chem. Lett. 2001, 12, 763.

12. Castagnolo, D.; Radi, M.; Dessì, F.; Manetti, F.; Saddi, M.; Meleddu, R.; De Logu, A.; Botta, M. Bioorg. Med. Chem. Lett. 2009, 19, 2203. 
13. Appukkuttan, P.; Dehaen, W.; Fokin, V. V.; Van der Eycken, E. Org. Lett. 2004, 6, 4223.

14. Feldman, A. K.; Colasson, B.; Fokin, V. V. Org. Lett. 2004, 6, 3897.

15. Scriven, E. F. V.; Turnbull, K. Chem. Rev. 1988, $2,351$.

16. (a) Girard, C.; Nen, E.; Aufort, M.; Beauvire, S.; Samson, E.; Herscovici, J. Org. Lett. 2006, 8, 1689. (b) Aufort, M.; Herscovici, J.; Bouhours, P.; Moreau, N.; Girard, C. Bioorg. Med. Chem. Lett. 2008, 18, 1195.

17. (a) Wiley, R.; Smith, N. R.; Johnson, D. M.; Moffat, J. J. Am. Chem. Soc. 1955, 77, 3412. (b) Labbe, G.; Bruynseels, M. J. Chem. Soc. Perkin Trans. 1 1990, 1492. (c) Labbe, G.; Bruynseels, M.; Delbeke, P.; Toppet, S. J. Het. Chem. 1990, 27, 2021. (d) Komanduri, V.; Krische, M. J. J. Am. Chem. Soc. 2006, 128, 16448.

18. De las Heras, F. G.; Alonso, R.; Alonso, G. J. Med. Chem. 1979, 22, 496.

19. (a) Lipshutz, B. H.; Taft, B. R. Angew. Chem. Int. Ed. 2006, 45, 8235. (b) Sreedhar, B.; Reddy, P. S. Synthetic Commun. 2007, 37,805.

20. Chassaing, S.; Sido, A. S. S.; Alix, A.; Kumarraja, M.; Pale, P.; Sommer, J. Chem. Eur. J. 2008, 14, 6713.

21. Durden, J.; Stansbury, H. A.; Catlette, W. H. J. Chem. Eng. Data 1964, 9, 228.

22. Namitharan, K.; Kumarraja, M.; Pitchumani, K. Chem. Eur. J. 2009, 15, 2755.

23. Smith, C. D.; Baxendale, I. R.; Lanners, S.; Hayward, J. J.; Smith, S. C.; Ley, S. V. Org. Biomol. Chem. 2007, 5, 1559.

24. (a) Moulin, F. Helv. Chim. Acta 1952, 35, 167. (b) Hagiwara, H.; Sasaki, H.; Hoshi, T.; Suzuki, T. Synlett 2009, 4, 643.

25. (a) Park, I. S.; Kwon, M. S.; Kim, Y.; Lee, J. S.; Park, J. Org. Lett. 2008, 10, 49. (b) Chan, T. R.; Hilgraf, R.; Sharpless, K. B.; Fokin, V. V. Org. Lett. 2004, 6, 2853. 